\title{
Pitch aircraft control with type-2 FLC and PD controller
}

\author{
Syed Mohamad Reza Haji Mirzaie ${ }^{1, ~ *, ~ H o d e i s e h ~ G o r d a n ², ~ J a l i l ~ S h i r a z i ~}{ }^{3}$, Amin Nikbakht ${ }^{1}$ \\ ${ }^{1}$ Electrical Engineering Department, Khavaran Institute of Higher Education, Mashhad, Iran \\ ${ }^{2}$ Department of Electrical Engineering, Sobhan Institute of Higher Education, Neyshabur, Iran \\ ${ }^{3}$ Department of Electrical Engineering, Islamic Azad University, Gonabad Branch, Gonabad, Iran

\section{Email address:} \\ Mohamad.hajimirzaie@gmail.com (S. M. R. H. Mirzaie), Hodeisehgordan@gmail.com (H. Gordan), Shirazi_stu@yahoo.com (J. Shirazi), \\ aminikbakht@yahoo.com (A. Nikbakht)
}

\section{To cite this article:}

Syed Mohamad Reza Haji Mirzaie, Hodeiseh Gordan, Jalil Shirazi, Amin Nikbakht. Pitch Aircraft Control with Type-2 FLC and PD Controller. Journal of Electrical and Electronic Engineering. Special Issue: Research and Practices in Electrical and Electronic Engineering in Developing Countries. Vol. 3, No. 2-1, 2015, pp. 12-15. doi: 10.11648/j.jeee.s.2015030201.13

\begin{abstract}
Aircraft systems are inherently unstable systems. The equations governing the motion of an aircraft are a very complicated set of six non-linear coupled differential equations. The linearized equation around the operating point is simulated in simulink MATLAB software. Also, the linear part of the system of nonlinear equations is simulated in simulink MATLAB software. In this study, combinations of PD controllers with fuzzy controller in a unity feedback system has been employed. This paper gives a comparison between the two types of FLC type-1 and type- 2 in order to show the great effect of the new type of FLCs in reducing overshoot of the step response and improving the robustness of the system.
\end{abstract}

Keywords: PD Controller, Pitch Aircraft Angle, Type-1 Fuzzy Logic Controller, Type-2 Fuzzy Logic Controller

\section{Introduction}

For linear systems and some of non-linear systems, classic controllers such as PD and PID have been widely used in industrial control processes because of their simple structure and robust performance in a wide range of operating conditions. Several numerical approaches such as Fuzzy Logic Controller (FLC) algorithm and evolutionary algorithms have been used for the optimum design of PID controllers [1]. FLS constructed based on type-1 fuzzy systems (T1FS), referred to as T1FLS, have demonstrated their ability in many applications, especially for the control of complex nonlinear systems that are difficult to model analytically [2, 3]. However, researchers have shown that T1FLS have difficulty in modeling and minimizing the effect of uncertainties [4]. One reason for this is that T1FS are certain in the sense that for each input there is a crisp membership grade. T2FS, characterized by membership grades that are themselves fuzzy, were first introduced by Zadeh in 1975 to account for this problem [5]. However, there were some obstacles about the implementation of the T2FLSs on real world problems such as characterization of type-2 fuzzy sets, performing operations with type-2 fuzzy sets, inferencing with type-2 fuzzy sets and obtaining the defuzzified value from the output of a type- 2 inference engine.
Since the publication of Karnik and Mendel [2], in which they proposed new concepts to overcome the difficulties mentioned above the number of literatures regarding T2FLSs have increased rapidly [6, 7]. In Satish et. al, [8], a new hybrid Fuzzy PD+ I controller $(\mathrm{FPD}+\mathrm{I})$ has been proposed and implemented.

In this paper, a pitch displacement of aircraft has been controlled and shows that the T2FL and PD controller with respect to T1FLC and $\mathrm{PD}$ controller improves the performance of a system.

This paper is organized as follows. Section 2 deals with the approximation of pitch aircraft control system model. In section 3, we present the analytical design of type-2 fuzzy control. Later, some simulations are executed to verify the validity of the proposed approach in section 4 . Finally, section 5 concludes the paper.

\section{Modeling a Pitch Aircraft Controller}

The active and influential forces on an aircraft are shown in the figure 1 . 


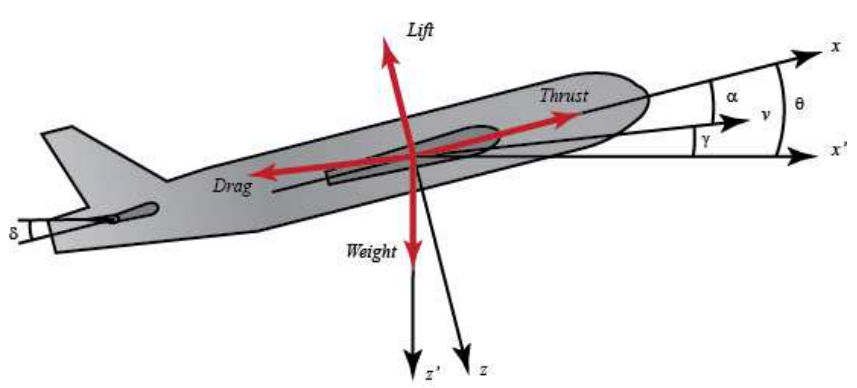

Figure 1. Forces acting on an aircraft.

The equations governing the motion of an aircraft are a very complicated set of six non-linear coupled differential equations. This article assumes that airplanes in the sky along the horizon is moving at constant speed and altitude. In this case, the resultant forces weight and lift and the lift and the resultant forces thrust where drag is equal to zero. Also, it is assumed that change in pitch angle does not change the speed of an aircraft. This assumption might seem unrealistic but simplifies the problem. Under these assumptions, the longitudinal equations of motion of an aircraft can be written as:

$$
\begin{gathered}
\dot{\alpha}=\mu \Omega \sigma\left[-\left(C_{L}+C_{D}\right) \alpha+\left(1 /\left(\mu-C_{L}\right)\right) q-\left(C_{w} \sin \gamma_{e}\right) \theta+C_{L}\right] \\
q=\mu \Omega / 2 i_{y y}\left\{\left[C_{M}-\eta\left(C_{L}+C_{D}\right)\right] \alpha+\left[C_{M}+\sigma C_{M}(1-\right.\right. \\
\left.\left.\left.\left.\mu C_{L}\right)\right]+\left(\eta C_{\mathrm{w}} \operatorname{Sin} \gamma_{e}\right) \delta_{e}\right]\right\} \\
\dot{\alpha}=\Omega q
\end{gathered}
$$

With replaced some numerical values of a Boeing 777 in equation (1), (2) and (3) we reach the following equations [10].

$$
\begin{gathered}
\dot{\alpha}=-0.313 \alpha+56.7 q+0.232 \delta_{\mathrm{e}} \\
\dot{\mathrm{q}}=-0.0139 \alpha-0.0426 \mathrm{q}+0.0232 \delta_{\mathrm{e}} \\
\dot{\theta}=56.7 \mathrm{q}
\end{gathered}
$$

Where $\alpha, \theta, q$ and $\delta$, are attack of angle, pitch angle, rate of change of pitch angle and the elevator deflection angle, respectively. To model systems, three state variable for the system are considered and the matrices $\mathrm{A}$ and $\mathrm{B}$ was ob-tained, as following:

$$
\left[\begin{array}{c}
\dot{\alpha} \\
\dot{\mathrm{q}} \\
\dot{\theta}
\end{array}\right]=\left[\begin{array}{ccc}
-0.313 & 56.7 & 0 \\
-0.0139 & -0.042 & 0 \\
0 & 56.7 & 0
\end{array}\right]\left[\begin{array}{l}
\alpha \\
\mathrm{q} \\
\theta
\end{array}\right]+\left[\begin{array}{c}
0.232 \\
0.0232 \\
0
\end{array}\right]\left[\delta_{\mathrm{e}}\right]
$$

According to the output, matrices $\mathrm{C}$ and $\mathrm{D}$ derived as follows:

$$
y=\left[\begin{array}{lll}
0 & 0 & 1
\end{array}\right]\left[\begin{array}{l}
\alpha \\
q \\
\theta
\end{array}\right]+[0]\left[\delta_{e}\right]
$$

After few steps of algebra, you should obtain the following transfer function:

$$
\frac{\theta(\mathrm{s})}{\delta_{\mathrm{e}}(\mathrm{s})}=\frac{1.151 \mathrm{~s}+0.1774}{\mathrm{~s}^{3}+0.739 \mathrm{~s}^{2}+0.921 \mathrm{~s}}
$$

Figure 2 presents the open-loop system step of response [11].

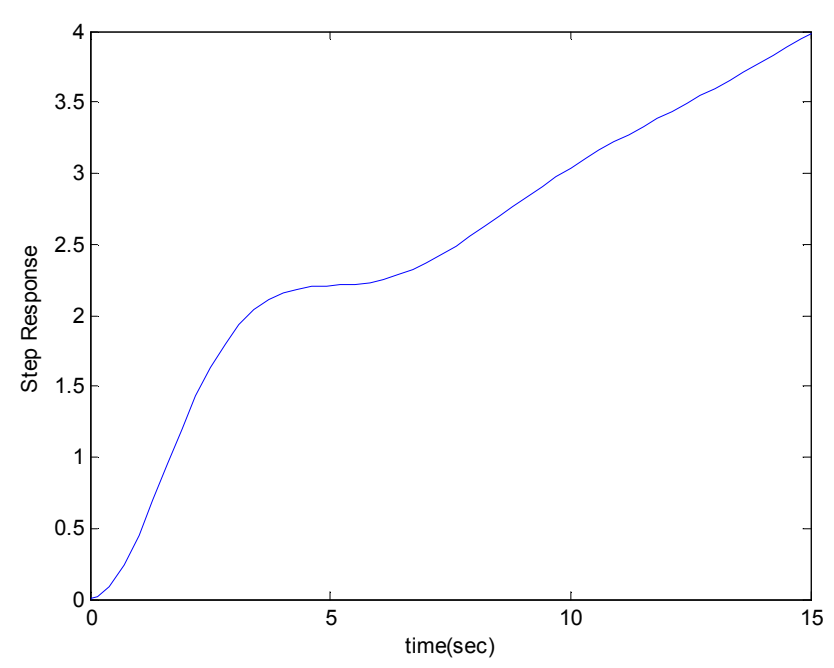

Figure 2. Open-loop step of response.

\section{Design Type-2 Fuzzy Logic Controller}

Fuzzy logic systems (FLCs) are based on the knowledge in the form of "IF-THEN" rules. Figure 3 shows a type-1 fuzzy logic system consisting of the fuzzifier and the defuzzifier. As illustrated in figure 3, a type-1 fuzzy logic system consists of 4 components: the rule base, the fuzzy inference engine, the fuzzifier and the defuzzifier [4]. In the type-1 fuzzy logic systems, for every input in the type- 1 collection of fuzzy there is degrees of membership with real recently, a type of fuzzy sets characterized by membership grades that are themselves fuzzy has been introduced. This fuzzy logic systems is type-2 fuzzy logic systems [4].

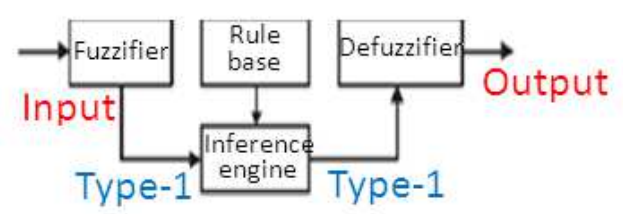

Figure 3. The main structure of type-1 fuzzy logic system.

T2 FLSs are characterized by the shape of their MFs. The membership function of general type-2 fuzzy is shown in figure 4 [6]. The extra mathematical dimension is provided by the blurred area. The FOU is bounded by upper and lower MFs.

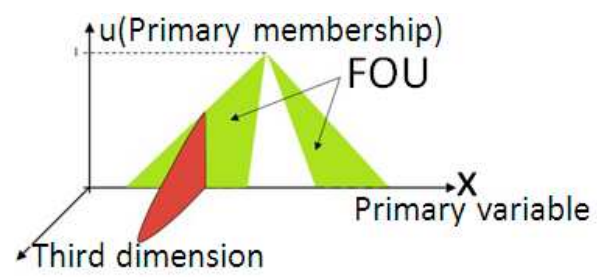

Figure 4. Type-2 fuzzy set.

The structure of a type-2 FLS is shown in figure 5 [4]. 
This structure is very similar to the type-1 fuzzy systems. Since the main difference between type 1 and type 2 fuzzy systems is the fuzzy sets. The only difference between type-1 and type-2 fuzzy logic system structure is in the block "type-reducer" .

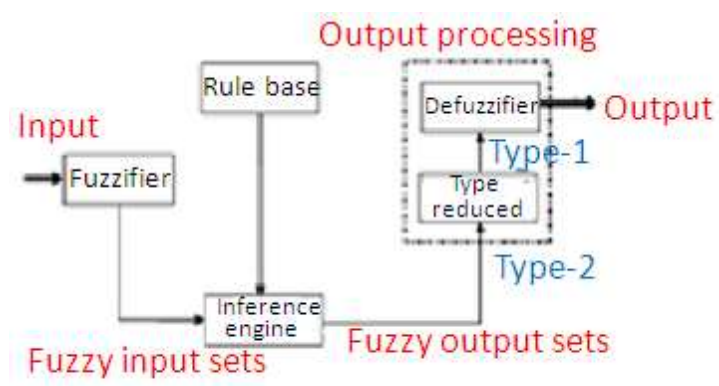

Figure 5. The main Structure of a T2 FLS

Each block is introduced in the following section:

\subsection{Fuzzifier}

The fuzzifier maps the input variables with the actual values to a type-1 or type-2 fuzzy set or a singleton fuzzy set in input.

\subsection{Rule base}

Fuzzy rule base is a set of fuzzy "if - then" rules. I-th law type 2 fuzzy systems is shown as follow:

$\mathrm{R}^{\mathrm{i}}$ : IF $\mathrm{x}_{1}$ is $\widetilde{\mathrm{F}}_{1}^{\mathrm{i}}$ and $\mathrm{x}_{2}$ is $\tilde{\mathrm{F}}_{2}^{\mathrm{i}}$ and $\ldots$ and $\mathrm{x}_{\mathrm{n}}$ is $\tilde{\mathrm{F}}_{\mathrm{n}}^{\mathrm{i}}$,

$$
\text { Then } y \text { is } \widetilde{G}^{\mathrm{i}}
$$

Where $\mathrm{i}=1, \ldots, \mathrm{M}$ and $\mathrm{N}$ is the number of rules. where $" \mathrm{~N}$ " implies that the fuzzy set is a type-2 fuzzy set. Where $x_{j}$ s are inputs $j=1, \ldots, n, \widetilde{F}_{j}^{i}$ is are input sets, $\widetilde{G}^{i}$ s are output sets and $\mathrm{y}$ is the output.

\subsection{Fuzzy Inference Engine}

The inference engine of a type-1 FLS provides a mapping from input type-1 fuzzy sets to output type-1fuzzy sets by using all rules. The inference process in a type- 2 FLS is very similar to that in a type-1 FLS. Hence a type-2 fuzzy member function mapping from input space to output space based on type- 2 fuzzy logic is created.

\subsection{Type-reduced}

In a type-1 FLS, the output of the inference engine is normally a type- 1 fuzzy set but, in a type- 2 FLS, the output of the inference engine is normally a type-2 fuzzy set. Hence, using a type-reduced block a type-1 fuzzy set is obtained from the type- 2 output sets of the FLS. This operation is called type reduction.

\subsection{Defuzzification}

In the defuzzification, a type-1 fuzzy set is produced to a crisp output.

\section{Simulation and Results}

The main goal of this study is to investigate and see whether the T2FLC performs better than similar T1FLC in controling the system. Therefore, in both cases we consider the same situation. The controller is designed mamdani type that in the number of rules and fuzzy sets for input and output are the same. Block diagram of the control structure using T2FLC and control of PD in the figure 6 is shown. In this paper, the parameters of $P D$ controlling is set with trial that $k_{P}$ and $k_{d}$ are considered 25 and 4 , respectively.

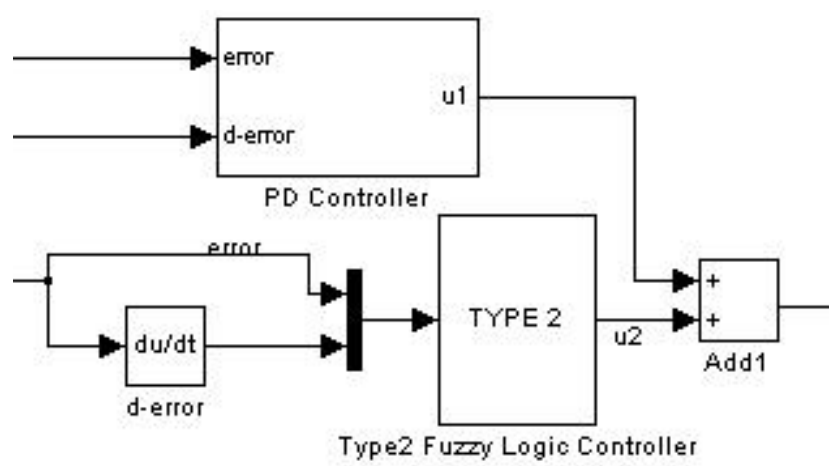

Figure 6. Block diagram of controller structure

In this study, the fuzzy controller has two inputs and one output. The error e $(\mathrm{t})$ and the change of error $((\mathrm{de}(\mathrm{t})) / \mathrm{dt})$ signals are used To design the fuzzy controller. In type 1 and type 2 fuzzy controller, gaussian functions are used as the input and output. Input variable are marked with three belong with the label of $\mathrm{N}, \mathrm{Z}$ and $\mathrm{P}$ and output variables are marked with the label GN , N , Z , P , GP GN. All the membership functions of the two FLCs inputs and outputs are shown in figures $7,8,9$, and 10 .

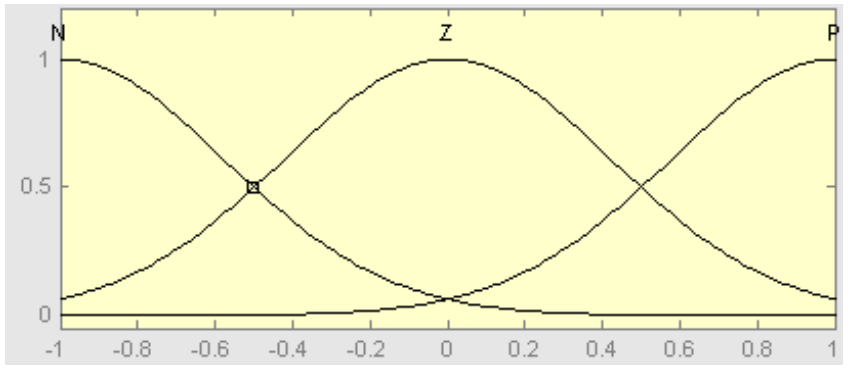

Figure 7. Type-1 fuzzy sets for the input variables.

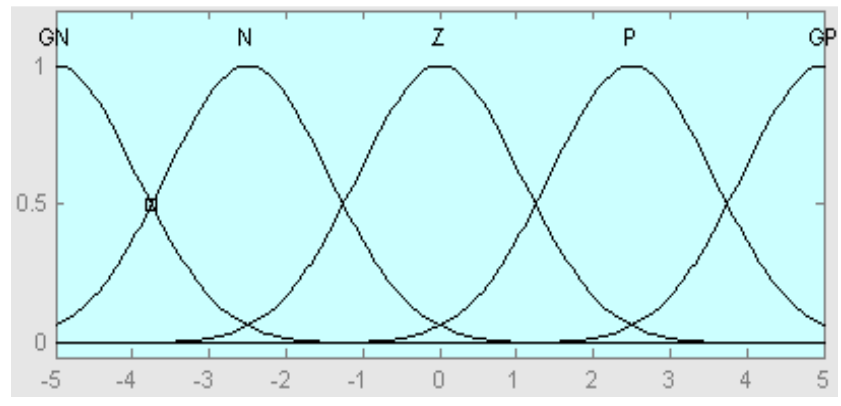

Figure 8. Type-1 fuzzy sets for the output variable. 


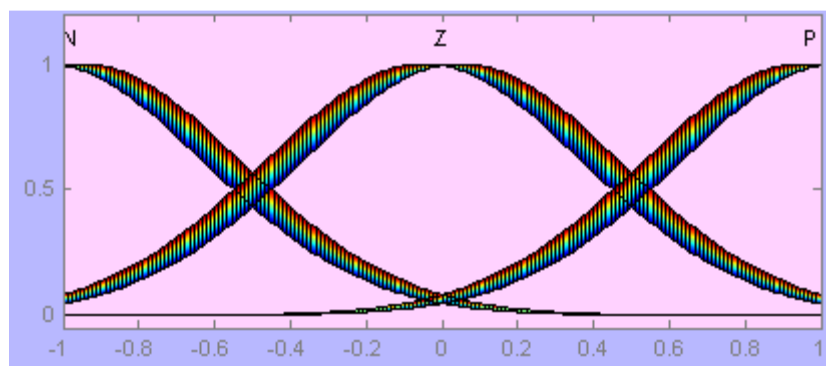

Figure 9. Type-2 fuzzy sets for the input variables.

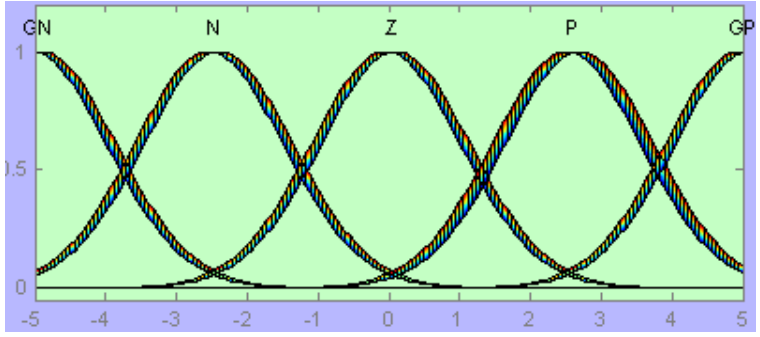

Figure 10. Type-2 fuzzy sets for the output variable.

Since we have considered for each input three fuzzy sets, we can evaluate the whole nine. All of the fuzzy rules collection are available in table 1 .

Table 1. Rule base of the two FLCs

\begin{tabular}{llll}
\hline d-error/error & $\mathbf{N}$ & $\mathbf{Z}$ & $\mathbf{P}$ \\
\hline $\mathbf{N}$ & $\mathrm{GN}$ & $\mathrm{N}$ & $\mathrm{Z}$ \\
$\mathbf{Z}$ & $\mathrm{N}$ & $\mathrm{Z}$ & $\mathrm{P}$ \\
$\mathbf{P}$ & $\mathrm{Z}$ & $\mathrm{P}$ & $\mathrm{GP}$ \\
\hline
\end{tabular}

In the fuzzy controller type -1 centroid method for defuzzification mechanism and in type-2 fuzzy controller to reduce the type of center method and center of gravity method for defuzzification is used for building. These methods using toolbox fuzzy type 2 is selected in the MATLAB environment [9]. Results are depicted in figure 11 and a comparison between the two types of FLCs and PD controller is done. The properties of each controller is shown in table 2 .

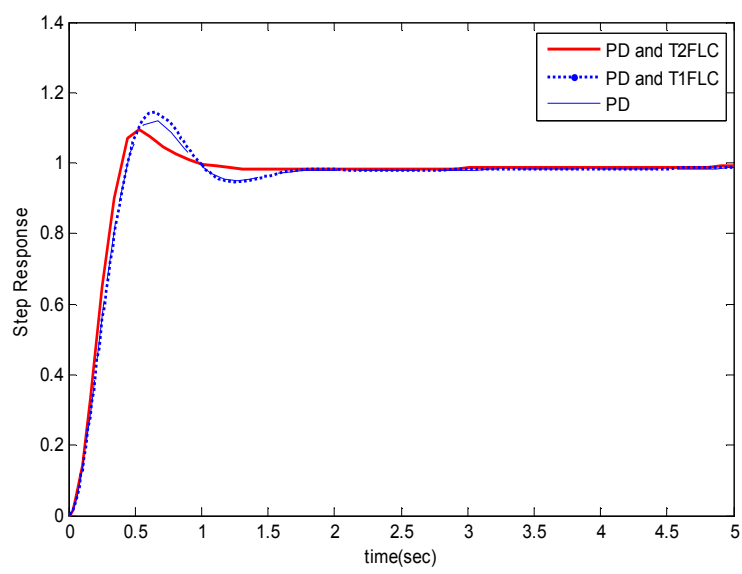

Figure 11. System response with T1FLC and T2FLC
Table 2. The properties of each controller

\begin{tabular}{lllll}
\hline $\boldsymbol{M}_{\boldsymbol{P}} \%$ & $\boldsymbol{t}_{\boldsymbol{p}}$ & $\boldsymbol{t}_{\mathbf{r}}$ & $\boldsymbol{t}_{\boldsymbol{s}}$ & Properties Controller \\
\hline $12 \%$ & 0.6675 & 0.4514 & 1.76 & Controller PD \\
$14.6 \%$ & 0.6562 & 0.445 & 1.7 & Controller PD and T1FLC \\
$9.4 \%$ & 0.5338 & 0.401 & 1.5 & Controller PD and T2FLC \\
\hline
\end{tabular}

\section{Discussion and Conclusion}

In this study, a PD controller with fuzzy controller is used. Fuzzy controllers are compared in two states: type 1 and type 2. As mentioned in table 2, we can conclude type-1 fuzzy controller does not always improve response. PD controller with the type- 2 fuzzy controller percent of rate overshoot step response other than the controller reduces. Also, we can conclude that, with given the uncertainty of fuzzy functions, the output response of the system is robust. These results are implemented using the IT2FLS toolbox in MATLAB software environment.

\section{References}

[1] Y. John and R. Langari, Fuzzy Logic Intelligence, Control and Information. NJ: Prentice Hall, 1998.

[2] P. King and E. Mamdani, The application of fuzzy control to industrial process, Automatica, vol. 13, pp. 235-242, 1997.

[3] L. A. Zadeh, Outline of a new approach to analysis of complex systems and decision processes. IEEE Trans. Syst., Man, Cybern., vol. 3, no. 1, pp. 28-44, 1973.

[4] J. M. Mendel, Rule-Based Fuzzy Logic Systems: Introduction and New Directions. NJ: Prentice-Hall, 2001.

[5] Zadeh, L. A., The concept of a linguistic variable and its application to approximate reasoning, Part I. Information Sciences, 1975, 8(3), pp. 199-249.

[6] Saba T. Salim, Design of Type-2 Fuzzy Logic Controller for a Simple Furnace System. Eng. \& Tech. Journal, Vol.30 , No. 8, 2012

[7] Ismail ATACAK, Design of a hybrid type-2 fuzzy logic/proportional integral controller for single-phase three-wire inverter system. Scientific Research and Essays Vol. 6(23), pp. 5049-5064, 16 October, 2011

[8] Satish. R. Vaishnav, Zafar. J. Khan, Performance of tuned PID controller and a new hybrid fuzzy PD + I controller, World Journal of Modelling and Simulation, Vol. 6, No. 2, pp. 141-149, 2010.

[9] M. B OZEK, Z. H. Akpolat, A Software Tool: Type-2 Fuzzy Logic Toolbox. Wiley Periodicals Inc. 2008.

[10] A. Torabi, A. Karsaz and S. K. M. Mashhadi, "Design of Modern and Predictive Controllers for Aircraft Control System", Latest Trends in Circuits, Systems, Signal Processing and Automatic Control, pp. 344-348, 2014.

[11] http://ctms.engin.umich.edu/CTMS/index.php?example=Aircr aftPitch\&section=SystemModeling 